\title{
FAT score: an Indian insight to a novel diagnostic score to differentiate non- alcoholic steatohepatitis (NASH) from simple steatosis
}

\author{
Jijo Varghese, Anoop K V*, Krishnadas Devadas and Tharun Tom
}

\begin{abstract}
Background: The aim of this study was to propose a simple predictive score to differentiate NASH from simple steatosis.

Results: This study included 64 patients who had biopsy-proven NAFLD, of which 34 patients had steatohepatitis and 30 had simple steatosis. Clinical, anthropometric, and biochemical variables of the study population were analyzed. Univariate analysis showed platelet count, ferritin, and transaminases (ALT\&AST) were predictors of NASH. This led to the proposal of a new diagnostic tool, FAT score ( $F$ signifies Ferritin, A indicates AST\&ALT, T denotes $t$ in Platelet) with AUROC of 0.95. The ROC curves for the significant variables were plotted and cutoff values were identified. Each component is awarded a score of 0 or 1 , based on this cutoff value. The component is awarded a score of 1 if the component score is above the cutoff value and 0 , if the score is below cutoff. The maximum score which can be obtained is 4 . A score of $\geq 3$ was able to predict NASH from simple steatosis with a sensitivity of $76.5 \%$ and a specificity of $100 \%$. The score was validated with a cohort of 84 liver biopsy patients wherein a cutoff $\geq 3$ was found to give a specificity of $100 \%$ in the validation cohort.

Conclusions: FAT score is a simple predictive model to differentiate NASH from simple steatosis (cutoff of more than or equal to 3) without performing a liver biopsy. A FAT score less than 3 rules out the need for biopsy.
\end{abstract}

Keywords: Fibrosis, Biopsy, Platelet count, Ferritin, Transaminases

\section{Background}

Non-alcoholic fatty liver disease (NAFLD) has become the most common cause of chronic liver disease. It is one of the most common causes of liver cirrhosis and liver transplantation. NAFLD is defined as steatosis $>5 \%$ in the absence of excessive alcohol consumption $(<20 \mathrm{~g} /$ day for females and $<30 \mathrm{~g} /$ day for males) [1]. The spectrum of NAFLD includes simple steatosis, nonalcoholic steatohepatitis (NASH), advanced fibrosis, and cirrhosis. NAFLD has many associations like metabolic syndrome, obesity, diabetes mellitus, dyslipidemia, and

* Correspondence: anoop85kv@yahoo.com

Government Medical College Thiruvananthapuram, Thiruvananthapuram, India insulin resistance [2]. Among the NAFLD spectrum, simple steatosis and NASH constitute the early form of the disease. A major cause of mortality in simple steatosis is cardiovascular disease. In the case of NASH, in addition to cardiovascular mortality, patients can progress to advanced fibrosis and cirrhosis and can result in liver-related mortality.

The prevalence of NAFLD is around 25.4\% worldwide (highest in the Middle East and lowest in Africa). Those with a BMI more than 30 have higher prevalence of NAFLD (90\%) when compared to those with BMI $<25$ (25\%) [3].

There are numerous non-invasive scoring systems like FIB-4, NFS score (NAFLD fibrosis score), BARD score,

\section{Springer Open}

(c) The Author(s). 2021 Open Access This article is licensed under a Creative Commons Attribution 4.0 International License, which permits use, sharing, adaptation, distribution and reproduction in any medium or format, as long as you give appropriate credit to the original author(s) and the source, provide a link to the Creative Commons licence, and indicate if changes were made. The images or other third party material in this article are included in the article's Creative Commons licence, unless indicated otherwise in a credit line to the material. If material is not included in the article's Creative Commons licence and your intended use is not permitted by statutory regulation or exceeds the permitted use, you will need to obtain permission directly from the copyright holder. To view a copy of this licence, visit http://creativecommons.org/licenses/by/4.0/. 
enhanced liver fibrosis score, and European liver fibrosis to diagnose advanced fibrosis which have a very good sensitivity and specificity. Among the NAFLD spectrum of disease, NASH and simple steatosis constitute the early stages of the disease. Simple steatosis is a benign condition, whereas NASH can progress to cirrhosis. Hence, it is important to distinguish NASH from simple steatosis. The significance of this study lies in the fact that it can differentiate NASH from simple steatosis without invasive investigations like liver biopsy.

Among the currently available non-invasive scoring systems, HAIR score [4], NASH test [5], NASH Clinical Scoring System for Morbid obesity [6], and NAFIC score [7] are the most accepted in the differentiation of NASH from simple steatosis. These scores have been developed primarily on studies of the European and American populations and very few scores have been developed by studying the Asian population. NAFIC score [7] was developed based on a study on the Japanese population. But the study contains type IV collagen and fasting insulin as variables, and these biochemical markers are not routinely available in laboratories, especially in resource limited setups.

Radiological studies can detect steatosis, but are ineffective in estimating more important steatohepatitis and fibrosis. Study conducted by Deniz Güney Duman concluded that the spleen-minus-liver attenuation difference (DeltaS-LA derived from CT) may be a useful method to detect steatosis from a CT scan [8]. But there was no correlation between DeltaS-LA and hepatic inflammation and fibrosis. Hence, it is time for the development of a scoring method based on simple biochemical and clinical variables that can predict NASH from simple steatosis in NAFLD. This study intended to propose a simple predictive score to differentiate NASH from simple steatosis.

\section{What is the need for this study?}

- Most scoring systems which differentiate NASH from simple steatosis were conducted in patients with morbid obesity which does not account for the data of lean NASH patients.

- The rest contain laboratory variables like cytokeratin 18, type IV collagen, hyaluronic acid, etc. which are costly and not easily available.

- It is important to distinguish NASH from simple steatosis because NASH can progress to advanced fibrosis and cirrhosis.

\section{Methods}

This was a diagnostic accuracy cross-sectional study which was conducted in the Department of Medical Gastroenterology [BLINDED FOR PEER REVIEW] from
July 2017 to June 2019. Before doing a liver biopsy, the following workup was done to find out etiology of the liver disease.

- IgG total and ANA-IF to diagnose autoimmune hepatitis

- HBsAG and IgM/IgG HBc to diagnose occult and overt HBV infection

- Anti-HCV to diagnose HCV infection

- Ceruloplasmin level, KF ring by slit lamp, and 24-h urine copper (if ceruloplasmin is low) to diagnose Wilson disease

- S. ferritin and transferrin saturation to diagnose hemochromatosis

The following populations were excluded from the study:

- Females with alcohol intake $\geq 2$ standard drink (one standard drink is $12 \mathrm{~g}$ ) and males with alcohol intake $\geq 3$ standard drink were excluded from the study.

- Those patients taking drugs which are likely to cause secondary steatosis were excluded.

- Borderline NASH patients (NAS score 3-4) were excluded.

An abdominal ultrasound was performed prior to the inclusion in the study population, and all patients whose liver biopsy was suggestive of NAFLD were included in the study.

Indications for doing liver biopsy were:

- Patients with transaminitis who had other etiological work ups negative

- Advanced fibrosis (F4) (defined as transient elastography (TE) value $>10 \mathrm{kPa}$ )

- Positive auto-antibodies to distinguish between autoimmune hepatitis or NAFLD

- High ferritin level to determine the extent of liver injury and iron accumulation

- Competing etiologies with NAFLD were suspected

Liver biopsy was taken using 16G liver biopsy needle under ultrasound guidance. One core biopsy was taken with a length of $1-1.5 \mathrm{~cm}$. At least 8 portal tracts were considered as adequate biopsy specimen. All biopsies were analyzed by a single pathologist who had experience of more than 15 years in liver histopathology to avoid inter-observer variability.

All patients undergoing liver biopsy with biopsyproven NAFLD without competing etiologies were included in the study. Patients were grouped into those with NASH and without NASH by taking NAS score of 
$>5$ as cutoff for NASH. Clinical, anthropometric, and biochemical variables were assessed on the same day of biopsy to find out the statistically significant variables for NASH. Biochemical variables used were hemoglobin, total count, platelet, packed cell volume, mean corpuscular volume, total bilirubin, albumin, alanine aminotransferase, aspartate aminotransferase, alkaline phosphatase, total cholesterol, triacylglycerol, high density lipoprotein, low-density lipoprotein, fasting blood sugar, $\mathrm{HBa} 1 \mathrm{C}-$ glycated hemoglobin levels, ferritin, and ceruloplasmin. Clinical and anthropometric variables used were presence or absence of diabetes mellitus, hypertension, dyslipidemia, or coronary artery disease and BMI.

The primary objective of the study was to find out the significant simple biochemical variables in predicting NASH in NAFLD. The secondary objective was to propose a new scoring system for NASH.

The study complied with the guidelines for human studies and was conducted ethically in accordance with the World Medical Association Declaration of Helsinki. Human ethics committee approval was taken for the study with ID No: - HEC.No.05/24/2019/MCT from "Institutional Ethics Committee, Government Medical College Thiruvananthapuram".

\section{Results}

Sixty-four biopsy-proven NAFLD patients were included in the study. Out of 64 patients, 36 were males and 28 were females. Thirty-four patients were biopsy-proven NASH and 30 were biopsy proven simple steatosis (Fig. 1). In the study population, 49 patients were overweight, 11 were obese, and 4 patients had a normal BMI. Among the qualitative variables analyzed, no variables were significant in predicting advanced fibrosis (Table 1). Among the quantitative variables, SGOT, SGPT, ferritin, and platelet count were significant in predicting NASH from simple steatosis (Table 2). There was no significant difference based on the presence or absence of diabetes to differentiate $\mathrm{NASH}$ and simple steatosis.

\section{Derivation of new score (FAT score)}

ROC curve for significant variables with AUC values more than 0.8 (Fig. 2) were plotted and cutoff values were identified. Cutoff value for aspartate aminotransferase (AST) was 48.5, alanine aminotransferase (ALT) 68.5 , ferritin 285 , and that of platelet was 2.2. Based on these cutoffs, ferritin AST ALT platelet (FAT) score was proposed. Each component is awarded a score of 0 or 1 based on this cutoff value (Table 3). The component is awarded a score of 1 if the component score is above the cutoff value and 0 , if the score is below cutoff. The maximum score which can be obtained is 4 . The ROC curve for the new score was plotted and found to be 0.955 . At a cutoff of $\geq 3$ new score had a specificity of $100 \%$ and a sensitivity of $76.5 \%$ in diagnosing NASH and simple steatosis (Fig. 3) (Table 4).

\section{Validation of FAT score}

Sample size for validation cohort was calculated by the formula $4 P Q / D^{2} /$ Prevalence,

where $P$ is Sensitivity, $Q$ is $1-P$, and $D$ is $20 \%$ of $P$.

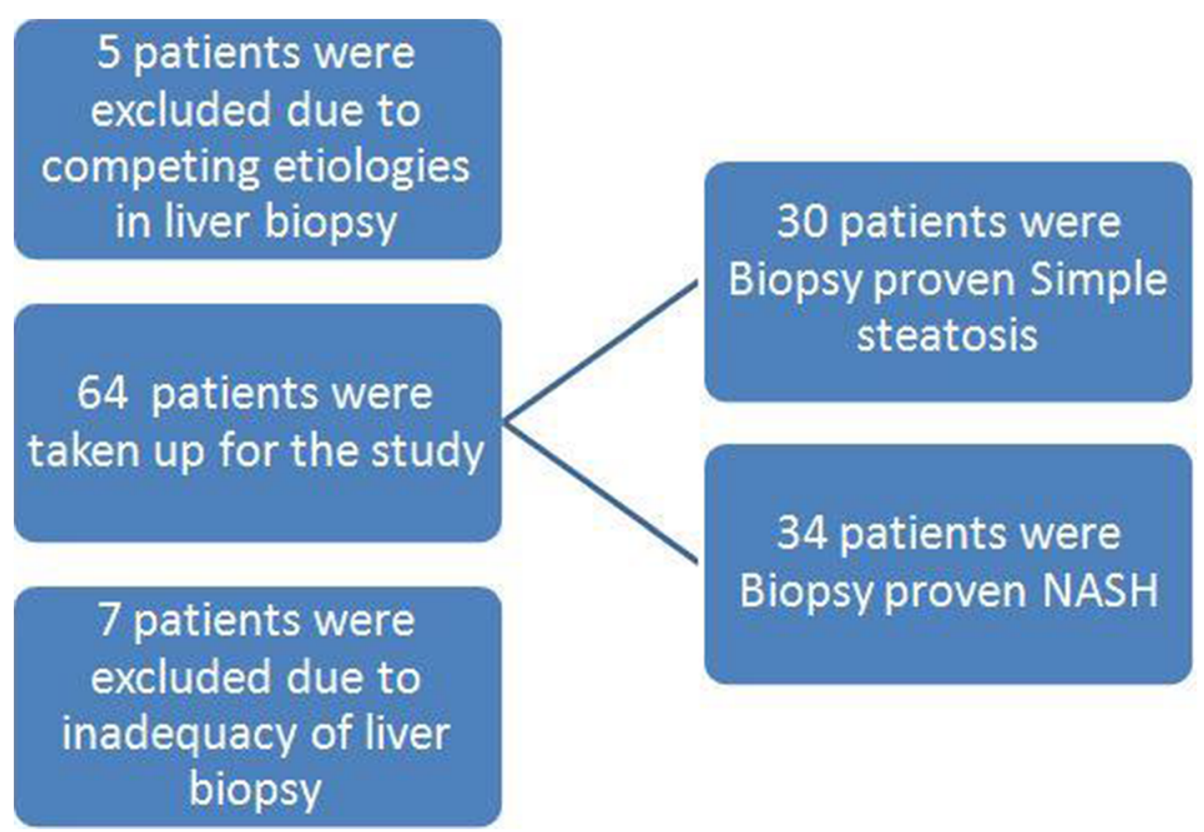

Fig. 1 Consort diagram for the study 
Table 1 Qualitative variables in predicting NASH from simple steatosis

\begin{tabular}{|c|c|c|c|c|c|c|c|}
\hline \multicolumn{8}{|c|}{ Table comparison of selected variables based on NASH } \\
\hline & & \multicolumn{4}{|l|}{ NASH } & \multirow[t]{3}{*}{$x^{2}$} & \multirow[t]{3}{*}{$p$} \\
\hline & & \multicolumn{2}{|l|}{ Present } & \multicolumn{2}{|l|}{ Absent } & & \\
\hline & & Count & Percent & Count & Percent & & \\
\hline \multirow[t]{2}{*}{ Sex } & Male & 20 & 55.6 & 16 & 44.4 & 0.1 & 0.755 \\
\hline & Female & 14 & 50.0 & 14 & 50.0 & & \\
\hline \multirow[t]{2}{*}{ DM } & Present & 16 & 72.7 & 6 & 27.3 & 2.59 & 0.108 \\
\hline & Absent & 18 & 42.9 & 24 & 57.1 & & \\
\hline \multirow[t]{2}{*}{ HTN } & Present & 14 & 53.8 & 12 & 46.2 & 0 & 0.946 \\
\hline & Absent & 20 & 52.6 & 18 & 47.4 & & \\
\hline \multirow[t]{2}{*}{ DLP } & Present & 20 & 47.6 & 22 & 52.4 & 0.74 & 0.388 \\
\hline & Absent & 14 & 63.6 & 8 & 36.4 & & \\
\hline \multirow[t]{2}{*}{$C A D$} & Present & 2 & 100.0 & 0 & 0.0 & 0.91 & 0.340 \\
\hline & Absent & 32 & 51.6 & 30 & 48.4 & & \\
\hline
\end{tabular}

$D M$ diabetes mellitus, HTN hypertension, DLP dyslipidemia, CAD coronary artery disease

Sensitivity of FAT score was $76.5 \%$ and prevalence of NASH in liver biopsy cohort was $53.125 \%$. Sample size for the validation cohort was calculated to be 58 . The study was validated in a liver biopsy cohort of 84 NAFLD patients. Inclusion criteria and methodology were similar to test cohort. In the validation cohort, the FAT score had area under the receiver operating characteristics (AUROC) of 0.893 in diagnosing NASH and simple steatosis (Fig. 4). At a cutoff of $>3$, the FAT score has a sensitivity of $75.6 \%$ and specificity of $100 \%$ in diagnosing NASH and simple steatosis in validation cohort (Table 5).

\section{Discussion}

A total 64 patients were taken up for the study out of which 34 were NASH patients and 30 were non-NASH simple steatosis patients. The study done by Dixon et al. to detect steatohepatitis had a sample size of 105 (HAIR score), 257 in the study by Poynard et al. (Nash test), 186 in Campos et al.'s study (NASH clinical scoring system), and 177 in Sumida et al.'s study (NAFIC score).

The HAIR score, which was initially introduced by Dixon JB et al., contained parameters like hypertension, ALT, and insulin resistance. A score of $\geq 2$ had high sensitivity and specificity for diagnosis of NASH. But HAIR score had poor sensitivity and specificity in diagnosing NASH in T2 diabetes mellitus (DM) patients [9]. Moreover, insulin resistance is not a commonly available test. FAT score had a good sensitivity and specificity in T2 DM patients.

NASH test was developed by Poynard et al., with the objective of diagnosing NASH in NAFLD patients. The score contained 13 parameters like alpha2macroglobulin, apolipoprotein A1, age, sex, height, weight, and serum levels of
Table 2 Quantitative variables in predicting NASH from simple steatosis

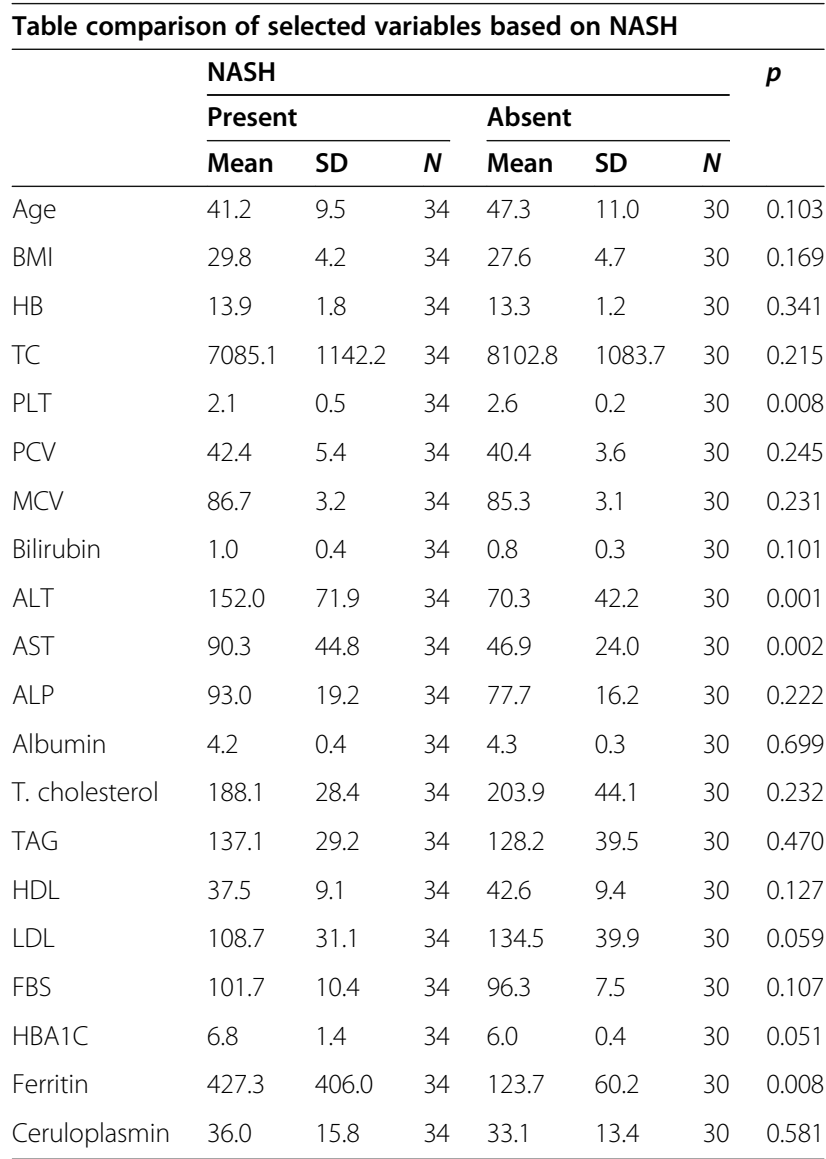

HB hemoglobin, TC total count, $P L T$ platelet, $P C V$ packed cell volume, $M C V$ mean corpuscular volume, ALT alanine aminotransferase, AST aspartate aminotransferase, ALP alkaline phosphatase, TAG triacylglycerol, HDL highdensity lipoprotein, $L D L$ low-density lipoprotein, $F B S$ fasting blood sugar, HBa1C glycated hemoglobin

triglycerides, cholesterol, transaminases ALT, AST, haptoglobin, gamma-glutamyl-transpeptidase, and total bilirubin. The score had several limitations like biopsy variability, limited inclusion criteria concerning alcohol consumption, etc. [5]. But the major advantage of FAT score over NASH test is the simplicity of FAT score in detecting steatohepatitis using commonly available bedside investigations, rather than parameters like alpha2macroglobulin, apolipoprotein A1, haptoglobin, etc.

The NASH clinical scoring system by Campos et al. detected steatohepatitis in morbidly obese patients (defined as body mass index $[\mathrm{BMI}]>35 \mathrm{~kg} / \mathrm{m}^{2}$ associated with obesity-related diseases or a BMI $>40$ with or without obesity-related diseases). The scoring system was able to identify high-risk patients who had NASH in morbidly obese patients [6]. But the capability of scoring system was limited to morbidly obese patients. The predominant study population in FAT score was overweight patients. Later, Sumida et al. proposed NAFIC score 


\section{ROC Curve}

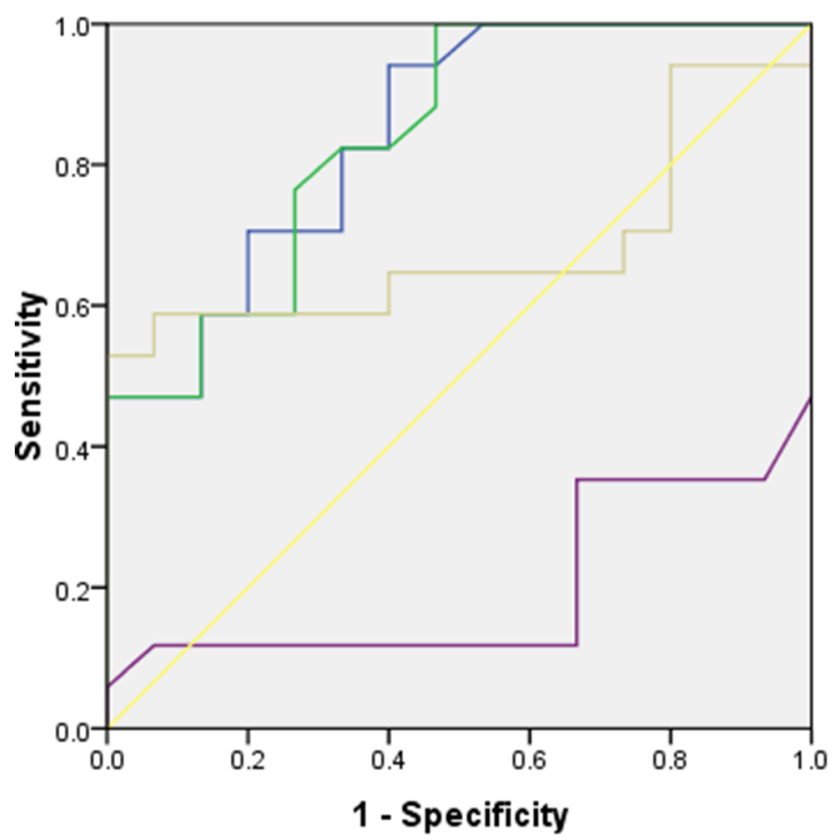

Source of the Curve

- SGOT

- SGPT

FERRITIN

- PLT

Reference Line

Diagonal segments are produced by ties.

Fig. 2 ROC curve of significant variables

which contained factors like serum ferritin, fasting insulin, and type IV collagen. The study excluded diabetic patients using exogenous insulin and insulin sensitizers, which is a major limitation of the study [7].

Most of the studies for NASH were done in morbid obesity patients, so it does not account for lean NASH patients. Many of these studies were done in western populations and had variables like cytokeratin 18 for evaluation. Such variables are costly and not routinely available in resource-limited areas. Our scoring system contains biochemical variables which are cheaply and routinely available in day to day practice.

The components which are used in FAT score for detecting steatohepatitis are common markers of liver injury. Ferritin, which is a marker of iron storage and also inflammation, can be elevated in NASH patients

Table 3 New score to differentiate NASH from steatosis (acronym is FAT score $\rightarrow$ F for ferritin A for AST and ALT and T for platelet)

\begin{tabular}{lll}
\hline Component & Score & Score \\
\hline Ferritin $(\geq 285$ was cut off $)$ & $0(<285)$ & $1(\geq 285)$ \\
AST $(\geq 49$ was cut off $)$ & $0(<49)$ & $1(\geq 49)$ \\
ALT $(\geq 69$ was cut off) & $0(<69)$ & $1(\geq 69)$ \\
Platelet $\leq 2.2$ lakh was cut off $)$ & $0(>2.2$ lakh $)$ & $1(\leq 2.2$ lakh $)$ \\
\hline
\end{tabular}

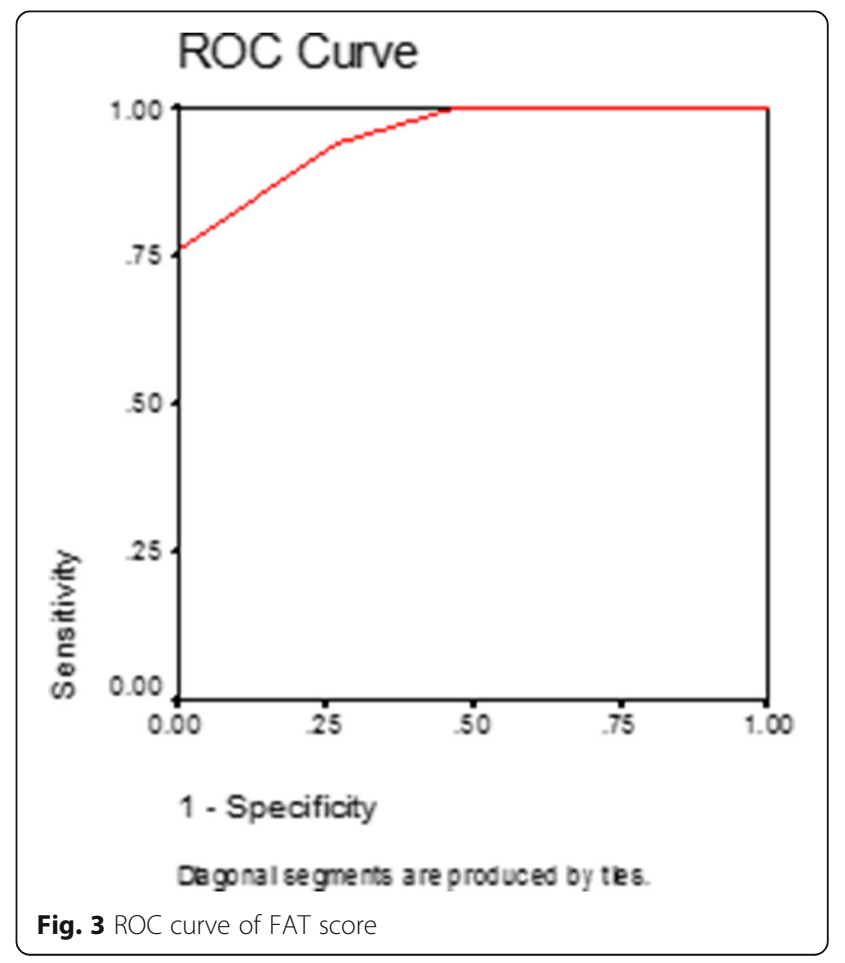


Table 4 Predictive power of FAT score in test cohort

\begin{tabular}{ll}
\hline Sensitivity & $\mathbf{7 6 . 5}$ \\
\hline Specificity & 100.0 \\
False negative & 23.5 \\
False positive & 0.0 \\
Positive predictive value & 100.0 \\
Negative predictive value & 78.9 \\
Negative likelihood ratio & 0.2 \\
Accuracy & 87.5 \\
\hline
\end{tabular}

[10]. NASH is a significant cause of secondary hemochromatosis. The study done in 628 patients by Kowdley KV et al. showed that a ferritin level more than 1.5 times the normal upper limit is associated with advanced fibrosis [11]. Thrombopoietin (TPO) which is a hormone produced in liver is utilized for platelet production. Thrombopoietin production can be affected by liver fibrosis. Weksler, in his, study tried to explore the etiology of thrombocytopenia in
Table 5 Predictive power of FAT score in validation cohort

\begin{tabular}{lll}
\hline Statistic & Value & $\mathbf{9 5 \%} \mathbf{C l}$ \\
\hline Sensitivity & $75.56 \%$ & $60.46 \%$ to $87.12 \%$ \\
Specificity & $100.00 \%$ & $90.97 \%$ to $100.00 \%$ \\
Negative likelihood ratio & 0.24 & 0.15 to 0.41 \\
Disease prevalence & $53.57 \%$ & $42.35 \%$ to $64.53 \%$ \\
Positive predictive value & $100.00 \%$ & \\
Negative predictive value & $78.00 \%$ & $67.96 \%$ to $85.56 \%$ \\
Accuracy & $86.90 \%$ & $77.78 \%$ to $93.28 \%$ \\
\hline
\end{tabular}

chronic liver disease patients and concluded that the development of thrombocytopenia in these patients is multifactorial [12]. Yoneda discovered a relationship between thrombocytopenia and the severity of liver injury [13]. There are currently numerous scoring systems in clinical use, which utilizes platelets as one of their components to diagnose liver injury like NAFLD fibrosis score, aspartate aminotransferase (AST)-toplatelet ratio index (APRI), and the FIB-4 index.

\section{ROC Curve}

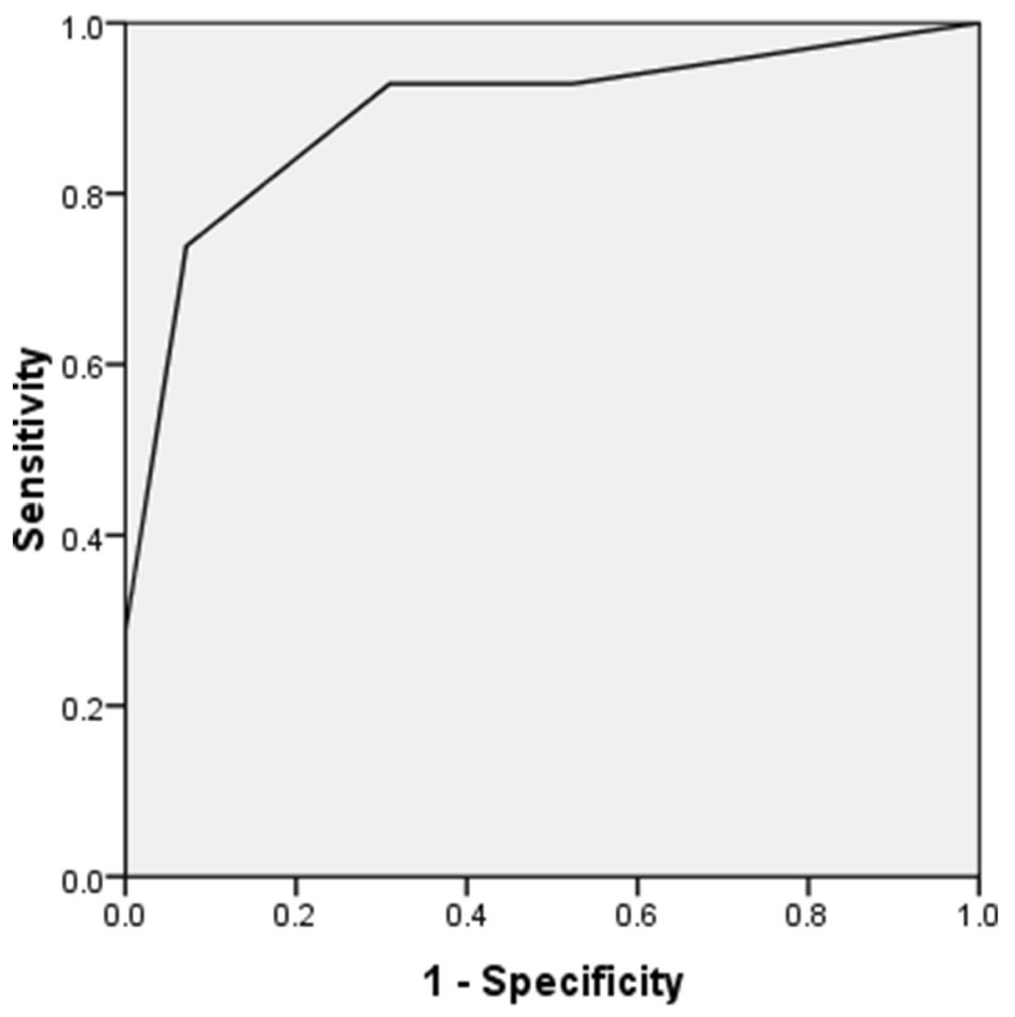

Diagonal segments are produced by ties.

Fig. 4 AUROC of FAT score in validation cohort 
AUROC of FAT score in predicting NASH from simple steatosis was 0.955 with sensitivity of $76 \%$ and specificity of $100 \%$ at a cutoff of $\geq 3$. AUROC of HAIR score was 0.9, that of Nash test was 0.78, NASH clinical scoring system had 0.8 , and 0.851 for NAFIC score in their original studies. FAT score has the highest AUROC in predicting NASH and simple steatosis in NAFLD.

FAT score was validated in a liver biopsy cohort of 84 patients. At a cutoff of $\geq 3$, the FAT score had a sensitivity of $75.6 \%$ and specificity of $100 \%$ in diagnosing NASH and simple steatosis in validation cohort. FAT score has a specificity of $100 \%$ in both test and validation cohort. It suggests that if FAT score is less than 3, unnecessary liver biopsies can be avoided, as the chance of NASH is $0 \%$ in such a situation.

Since the score was developed and validated in NAFL D cohort requiring liver biopsy, the reproducibility of the score in NAFLD patients who do not require liver biopsy needs to be assessed separately. As FAT score had specificity of $100 \%$ in test and validation cohort, it can circumvent the need for liver biopsy in patients with a FAT score $<3$.

One of the limitations of the study was that borderline NASH patients were excluded from the study. Since the number of lean NASH patients was limited, the difference in NASH patients based on BMI cannot be accurately measured. Further studies are required in lean NASH patients, before we can declare that FAT score has the same sensitivity and specificity in lean NASH patients to detect steatohepatitis as that of overweight and obese patients.

In conclusion, FAT score is a simple predictive model to differentiate NASH from non-NASH NAFLD or simple steatosis (cutoff of more than or equal to 3) without a liver biopsy, while giving a high sensitivity, specificity and accuracy. FAT score less than 3 rules out the need for a biopsy. It can be used as a screening tool instead of biopsy. However, a multi-center validation study is required to find out the reproducibility of FAT score.

\section{Abbreviations \\ ALT: Alanine aminotransferase; ALP: Alkaline phosphatase; AST: Aspartate aminotransferase; AUROC: Area under the receiver operating characteristics; CAD: Coronary artery disease; DM: Diabetes mellitus; DLP: Dyslipidemia; FAT score: Ferritin AST ALT Platelet score; FBS: Fasting blood sugar; HB: Hemoglobin; HBa1C: Glycated hemoglobin; HDL: High-density lipoprotein; HTN: Hypertension; LDL: Low-density lipoprotein; MCV: Mean corpuscular volume; NAFLD: Non-alcoholic fatty liver disease; NASH: Non- alcoholic steatohepatitis; PCV: Packed cell volume; TAG: Triacylglycerol; TC: Total count; TE: Transient elastography}

\section{Acknowledgements}

None.

\section{Authors' contributions}

The entire manuscript was prepared by JV, AKV, KD, and $T$. JV made contributions to conception and design of the work. AKV made contributions to the acquisition, analysis, and interpretation of data. KD made contributions to interpretation of data. $\Pi$ made contributions to revision of the work. All authors have read and approved the manuscript.

Funding

None.

Availability of data and materials

1. https://pubmed.ncbi.nlm.nih.gov/27188459/

2. https://www.ncbi.nlm.nih.gov/pmc/articles/PMC1657015/

\section{Declarations}

Ethics approval and consent to participate

Written consent was taken to participate in the study. Human ethics committee approval was taken for the study with ID No: - HEC.No.05/24/ 2019/MCT from "Institutional Ethics Committee, Government Medical College, Thiruvananthapuram".

\section{Consent for publication}

Obtaining informed consent to publish the information from the study participants: written consent was obtained to publish the study.

\section{Competing interests}

The authors declare that they have no competing interests.

Received: 6 July 2021 Accepted: 8 September 2021

Published online: 08 December 2021

References

1. Brunt EM, Wong WW, Nobili V, Day CP, Sookoian S et al (2015) Nonalcoholic fatty liver disease. Nat Rev Dis Primers 1(1):15080. https://doi.org/10.1038/ nrdp.2015.80

2. Eur. Assoc. Study Liver, Eur. Assoc Study Diabetes, Eur. Assoc. Study Obes (2016) EASL-EASDE ASO clinical practice guidelines for the management of non-alcoholic fatty liver disease. J Hepatol 64:1388-1402

3. Younossi ZM, Koenig AB, Abdelatif D, Fazel Y, Henry L, Wymer M (2016) Global epidemiology of nonalcoholic fatty liver disease-meta-analytic assessment of prevalence, incidence, and outcomes. Hepatology 64:73-84

4. Dixon JB, Bhathal PS, O'Brien PE (2001) Nonalcoholic fatty liver disease: predictors of nonalcoholic steatohepatitis and liver fibrosis in the severely obese. Gastroenterology 121(1):91-100. https://doi.org/10.1053/ gast.2001.25540

5. Poynard T, Ratziu V, Charlotte F, Messous D, Munteanu M, Imbert-Bismut F, Massard J, Bonyhay L, Tahiri M, Thabut D, Cadranel JF, Le Bail B, de Ledinghen V, LIDO Study Group, CYTOL study group (2006) Diagnostic value of biochemical markers (NashTest) for the prediction of nonalcoholic steatohepatitis in patients with non-alcoholic fatty liver disease. BMC Gastroenterol 6(1):34. https://doi.org/10.1186/1471-230X-6-34

6. Campos GM, Bambha K, Vittinghoff E, Rabl C, Posselt AM, Ciovica R, Tiwari U, Ferrel L, Pabst M, Bass NM, Merriman RB (2008) A clinical scoring system for predicting nonalcoholic steatohepatitis in morbidly obese patients. Hepatology 47(6):1916-1923. https://doi.org/10.1002/hep.22241

7. Sumida Y, Yoneda M, Hyogo H, Yamaguchi K, Ono M, Fujii H, Eguchi Y, Suzuki Y, Imai S, Kanemasa K, Fujita K, Chayama K, Yasui K, Saibara T, Kawada N, Fujimoto K, Kohgo Y, Okanoue T, Japan Study Group of Nonalcoholic Fatty Liver Disease (JSG-NAFLD) (2011) A simple clinical scoring system using ferritin, fasting insulin, and type IV collagen 7S for predicting steatohepatitis in nonalcoholic fatty liver disease. J Gastroenterol 46(2):257-268. https://doi.org/10.1007/s00535-010-0305-6

8. Duman DG, Celikel C, Tüney D, Imeryüz N, Avsar E, Tözün N (2006) Computed tomography in nonalcoholic fatty liver disease: a useful tool for hepatosteatosis assessment? Dig Dis Sci 51(2):346-351. 16534679. https:// doi.org/10.1007/s10620-006-3136-9

9. Angelidi AM, Papazafiropoulou AK, Tzouganatou E et al (2017) Evaluation of different scores to predict non-alcoholic fatty liver disease in patients with type 2 diabetes. Hell J Atheroscler 8:103-112

10. Shah RA, Kowdley KV (2019) Serum ferritin as a biomarker for NAFLD: ready for prime time? Hepatol Int 13(2):110-112. https://doi.org/10.1007/s12072-01 9-09934-7

11. Kowdley KV, Belt P, Wilson LA, Yeh MM, Neuschwander-Tetri BA, Chalasani N, Sanyal AJ, Nelson JE, for the NASH Clinical Research Network (2012) 
Serum ferritin is an independent predictor of histologic severity and advanced fibrosis in patients with nonalcoholic fatty liver disease. Hepatology 55(1):77-85. https://doi.org/10.1002/hep.24706

12. Weksler BB (2007) Review article: the pathophysiology of thrombocytopenia in hepatitis $C$ virus infection and chronic liver disease. Aliment Pharmacol Ther 26(Suppl 1):13-19. https://doi.org/10.1111/j.1365-2036.2007.03512.x

13. Yoneda M, Fujii H, Sumida Y, Hyogo H, Itoh Y, Ono M et al (2011) Platelet count for predicting fibrosis in nonalcoholic fatty liver disease. J

Gastroenterol 46(11):1300-1306. https://doi.org/10.1007/s00535-011-0436-4

\section{Publisher's Note}

Springer Nature remains neutral with regard to jurisdictional claims in published maps and institutional affiliations.

\section{Submit your manuscript to a SpringerOpen ${ }^{\circ}$ journal and benefit from:}

- Convenient online submission

- Rigorous peer review

- Open access: articles freely available online

- High visibility within the field

- Retaining the copyright to your article

Submit your next manuscript at $\boldsymbol{\nabla}$ springeropen.com 